Communications in Physics, Vol. 29, No. 3 (2019), pp. 215-221

DOI:10.15625/0868-3166/29/3/13759

\title{
APPLYING FIBER BRAGG GRATING FOR ACOUSTIC SENSOR
}

\author{
PARADORN PAKDEEVANICH ${ }^{\dagger}$, NATTAPON LIKITTANANAN \\ AND WORRAPASS PROMSEN
}

Department of Physics, Faculty of Science, Silpakorn University, Nakorn Prathom, Thailand

${ }^{\dagger} E$-mail: pakdeevanich_p@su.ac.th

Received 17 April 2019

Accepted for publication 28 June 2019

Published 15 August 2019

\begin{abstract}
In this work, we have interested to utilize Fiber Bragg Grating (FBG) for monitoring sound intensity level. Acoustic disturbance could induces train and changing the refractive index of FBG. Therefore, light will interfere for the trough of transmission at one specific wavelength satisfied Bragg's condition. In our demonstrations FBG sensor was perturbed with sound intensity levels of 60, 80 and $100 \mathrm{~dB}$, the measuring Bragg wavelengths were recorded at1552 $\mathrm{nm}, 1553$ $\mathrm{nm}$ and $1555 \mathrm{~nm}$,respectively. It was found that the Bragg wavelength shift has a quite linear variation with sound intensity level. In addition, the sensitivity for sensor was $0.0566 \mathrm{~nm} / \mathrm{dB}$. The obtained results are promising for developing acoustic and vibration sensors
\end{abstract}

Keywords: fiber-optic detection of sound, optical fibre sensors, fiber Bragg grating sensor, sound intensity sensor acoustic sensor.

Classification numbers: 42.81.Pa; 07.60.Vg.

(C)2019 Vietnam Academy of Science and Technology 


\section{INTRODUCTION}

In the last decade, tremendous amount of innovation in optical fiber sensors had been challenged to monitor some physical parameters namely temperature, pressure, acceleration, rotation, force, and vibration of structure. Because of many advantages including immunity towards electromagnetic interference, highly sensitive and compactness fiber optic sensors perform reliable and highly sensitive tasks over conventional electrical sensors Fiber-optic sensor works by modulating one or more properties of a propagating light wave, including intensity, phase, polarization, and frequency, in response to the environmental parameter being measured.

A rapid development has led research activity aimed to measure the sound intensity level or acoustic sensor. Fiber acoustic sensors play important roles in several applications such as oil exploration, sonar navigation, surveillance, magnetic resonance imaging, structural health monitoring and biological applications. The bare fibers have been used primarily but it performed low sensitivity. Later, the use of appropriate coating material has been investigated to improve sensitivity of the sensor. In general, those developements were concerned with the mechanism for conversion of perturbation to the change in the refractive index of cladding of the fiber. Generally, these mechanisms caused of the changes in optical power at the exit end of the fiber.

We have intended to propose a new design of high sensitivity optical fiber sensor for detection sound. Fiber Bragg Gratings (FBGs) are selected because of high sensitivity and large operational bandwidth. FBG-based sensors have been developed to observe the spectral response [1]. FBG is a distributed Bragg reflector constructed in a short segment of optical fiber. The periodic modulation of the refractive index in the core of an optical fiber is achieved by using an interference pattern of an intense ultraviolet laser light. When injecting a spectral broadband light into the fiber, the particular Bragg wavelength will be reflected by the inscribed grating. The Bragg's condition for the reflected waves interfered constructively is expressed as [1]

$$
\begin{aligned}
2\left(\frac{2 \pi n_{e f f}}{\lambda_{B}}\right) & =\frac{2 \pi}{\Lambda} \\
\lambda_{B} & =2 n_{\text {eff }}
\end{aligned}
$$

where $\lambda_{B}$ is the Bragg wavelength of FBG, $n_{\text {eff }}$ is the effective refractive index of the fiber and $\Lambda$ is the grating spacing or the pitch of the grating. The wavelength which satisfied the Bragg's condition is reflected back from the grating region and the rest is transmitted. Consequently, the reflected light achieves the peak at the Bragg wavelength. In parallel, the trough of transmission occurs at the Bragg wavelength as displayed in Fig. 1.

Theoretically, a longitudinal deformation due to an external perturbation may change both

$n_{\text {eff }}$ and $\Lambda$. The relation between the relative shift in Bragg wavelength of FBG, $\Delta \lambda_{B} / \lambda_{B}, n_{\text {eff }}$ and $\Lambda$ applied to the grating at constant temperature is given by the partial derivative of Eq. (2) with respect to displacement:

$$
\frac{\Delta \lambda_{B}}{\lambda_{B}}=2 n_{e f f} \frac{\partial \Lambda}{\partial L}+2 \Lambda \frac{\partial n_{e f f}}{\partial L}
$$

In our design sensor system, it was based on the hypothesis that the variation in sound intensity is capable of changing $n_{\text {eff }}$ and $\Lambda$ parameters. Essentially, applied sound will induce strain on FBG sensing area. Therefore, the relative shift in the Bragg wavelength $\left(\Delta \lambda_{B}\right)$ can be measured and it must be dependent on the level of sound intensity as simulated results in Fig. 2. 


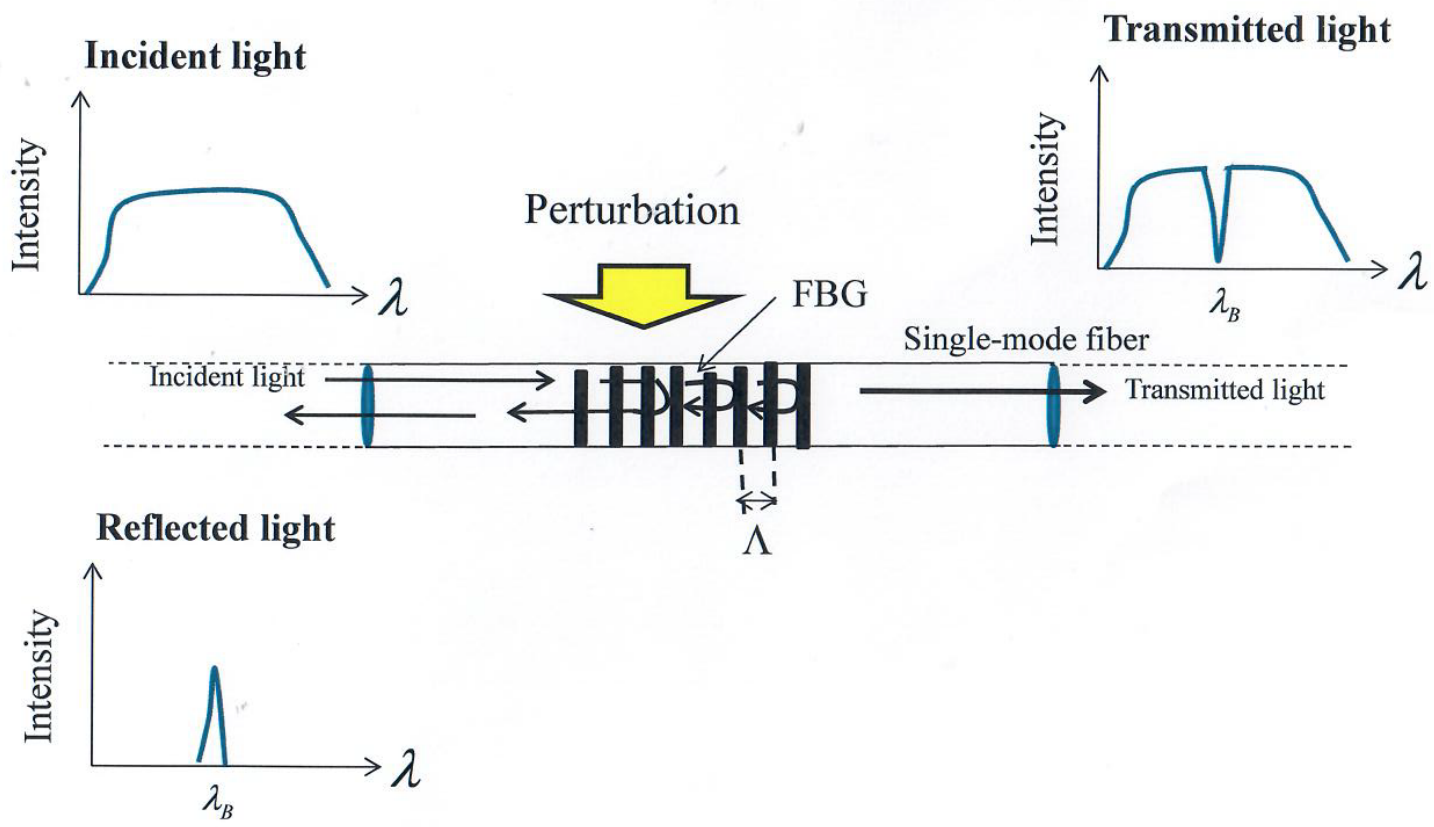

Fig. 1. Structure of sensor based on FBG and intensity for incident light, reflected light and transmitted light.

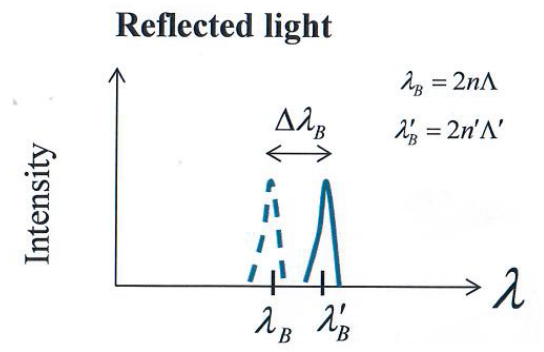

(a)

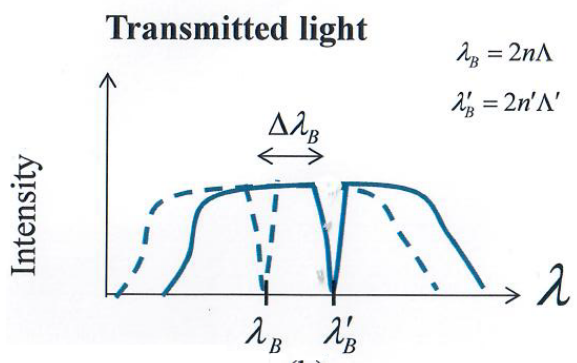

(b)

Fig. 2. The shift in the Bragg wavelength $\left(\Delta \lambda_{B}\right)$ for (a) reflected light and (b) transmitted light.

In previous works, H. L. Liu et al. [2] reported the experimental study on FBG strain sensor and the shifted Bragg wavelengths were monitored. C. Li et al. [3] suggested to use the sidepolished fiber Bragg grating to enhance the performance of optical fiber sensor. M. F. Liang et al. [4] installed FBG on the cantilever to construct pressure sensor and their innovative sensor could obtain a sensitivity about $339.956 \mathrm{pm} / \mathrm{Mpa}$. In addition, Z. Zhang et al. [5] employed FBG to calibrate the effect of temperature and pressure and use metal membrane to control the pressure. The accuracy of $0.1 \mathrm{hPa}$ in the range $800-1300 \mathrm{hPa}$ was presented. 


\section{EXPERIMENT}

In our design system, it consisted of a tunable semiconductor laser (SANTEC TSL-210), FBG (bandwidth wavelength of 1525-1565 $\mathrm{nm}$ ) and spectrum analyzer (ANDO AQ6317B) as shown photographs in Fig. 3. Loudspeaker was generated from Samsung Galaxy S7 Edge with sound generator application. IPhone 6 with sound analyzer application was utilized to monitor sound intensity level. The schematic diagram of the experimental setup was shown in Fig. 4. The power of optical light sources was fixed a constant power output at $3.2 \mathrm{~mW}$ (across all wavelengths) and connected the optical aperture with FBG. The wavelength of laser was tuned between $1530 \mathrm{~nm}$ to $1610 \mathrm{~nm}$ (increasing in step of $1 \mathrm{~nm}$ ). The output of FBG was connected to spectrum analyser. The frequency of loudspeaker was set constantly at $1000 \mathrm{~Hz}$ and operated the sound intensity level at 60,80 and $100 \mathrm{~dB}$. For each intensity level of sound, we performed and repeated experiment for 3 times. Optical output power and FBG wavelength shifts were measured and recorded by spectrum analyzer. Then, the obtained results were averaged. In the situation that operated the sensor without perturbation was served as the reference.

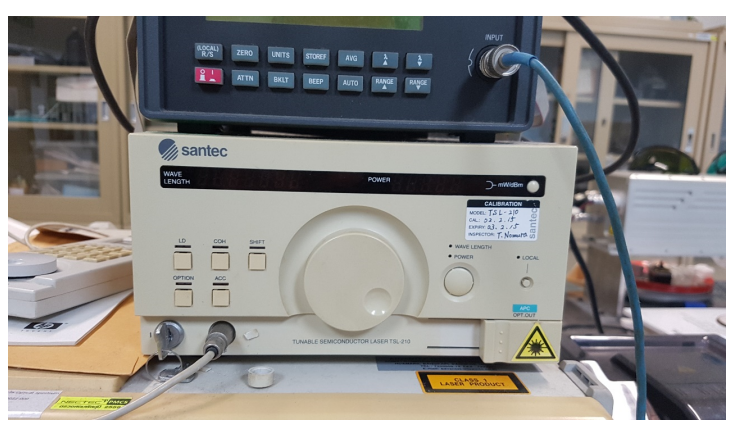

(a)

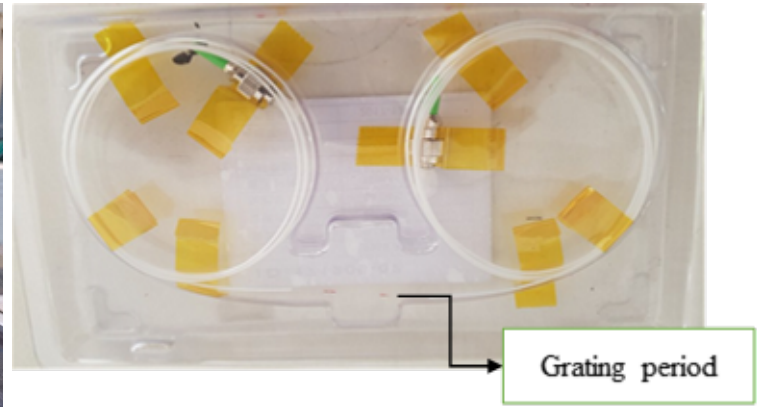

(b)

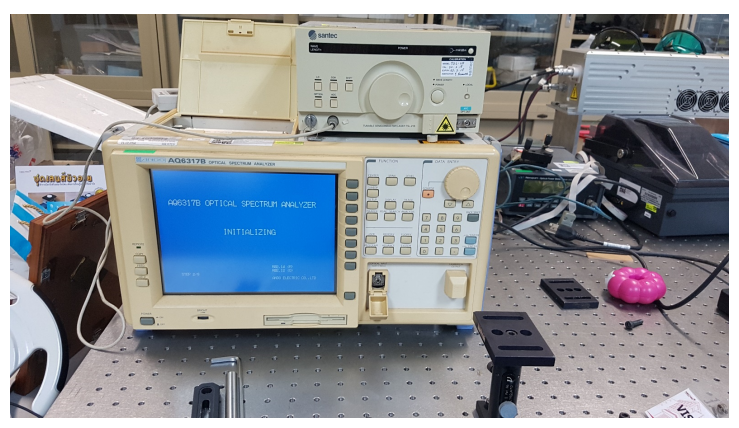

(c)

Fig. 3. The photographs of (a) tunable semiconductor laser, (b) single mode FBG and (c) spectrum analyzer. 


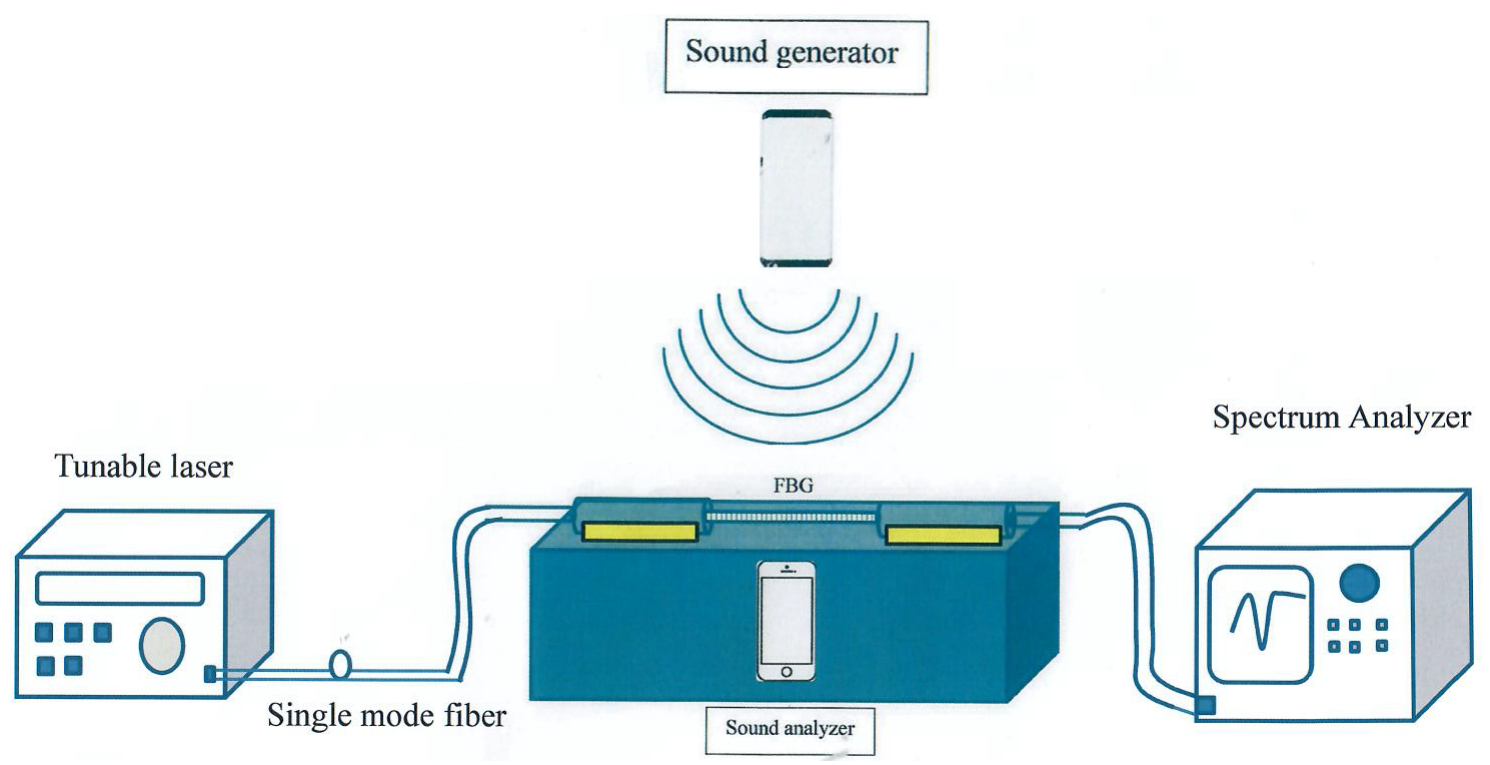

Fig. 4. The schematic diagram of the experimental setup.

\section{RESULTS AND DISCUSSION}

For undisturbing situation, the output spectrum showed the trough of transmission at the wavelength $1549 \mathrm{~nm}$. When generated sound to disturb FBG, the transmitted spectra for sound intensity level 60, 80 and $100 \mathrm{~dB}$ were obtained as presented in Fig. 5(a), 5(b) and 5(c), respectively.

The wavelength shift $\left(\Delta \lambda_{B}\right)$ for each sound intensity level $\left(I_{\text {sound }}\right)$ was monitored and plotted graph as shown in Fig. 6.

According to Fig. 6, we obtained the linear relationship between the wavelength shift $\left(\Delta \lambda_{B}\right)$ and the sound intensity level $\left(I_{\text {sound }}\right)$ as the following equation,

$$
\Delta \lambda_{B}=0.0566 I_{\text {sound }}-0.1726
$$

Therefore, we could determine the sensitivity of our sensor to be equal to $0.0566 \mathrm{~nm} / \mathrm{dB}$. However, we admitted that our experimental results lacked of studying in the stability of sound measurement in practice. It is necessary to investigate the stability of the FBG wavelength shifts since it strongly depend on other parameter of sound such as the direction of sound and the vibration frequency. To achieve a higher detection sensitivity, our future developments are attempted such as bending FBG, or FBG cladding substituted by the surrounding media, or combining with piezoelectric materials or using membrane attached on surface or vibration system aroud FBG or applying tilt FBG etc. 

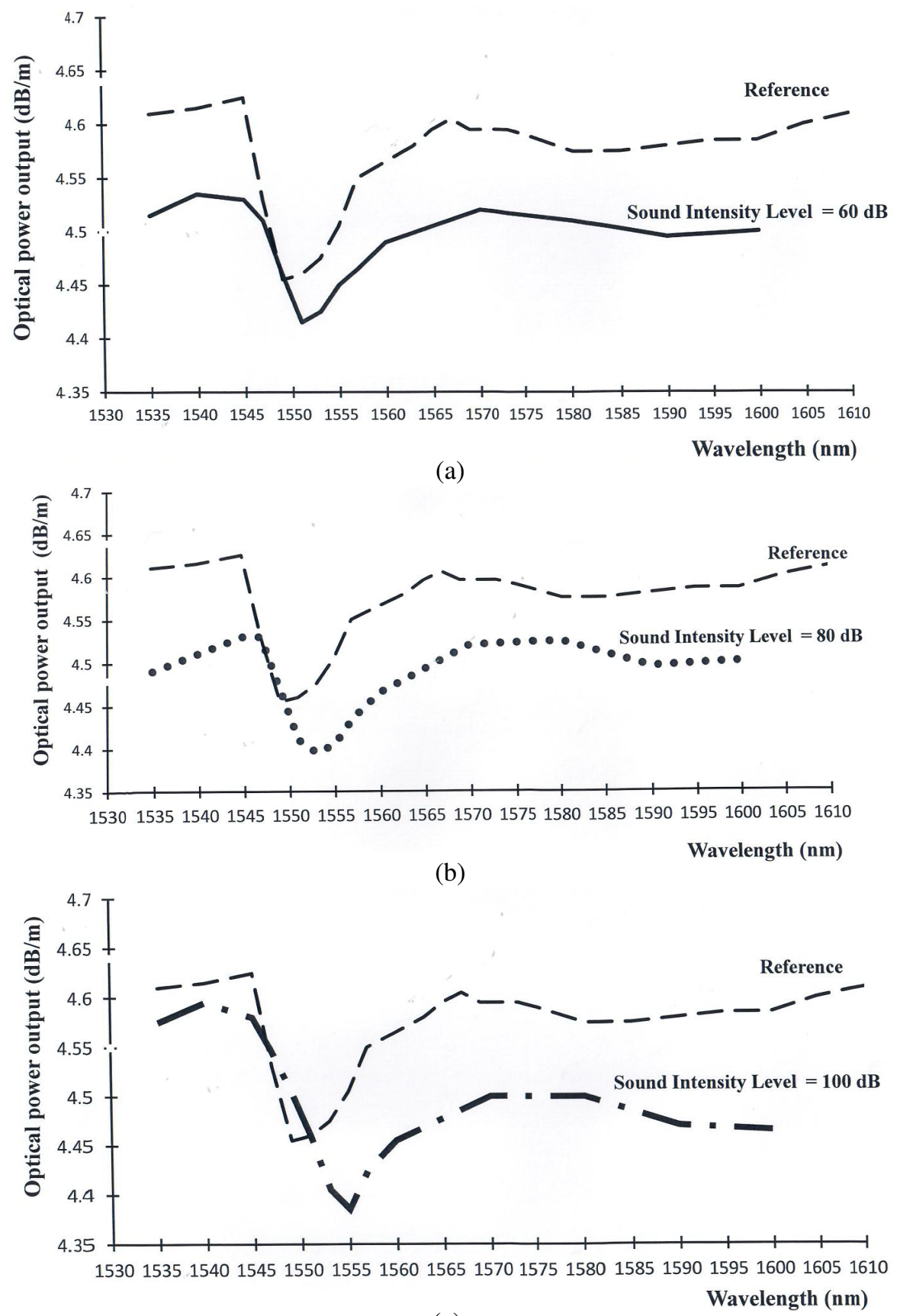

(c)

Fig. 5. Spectrum of transmission for disturbation FBG with various sound intensity levels: (a) $60 \mathrm{~dB}$, (b) $80 \mathrm{~dB}$ and (c) $100 \mathrm{~dB}$. 


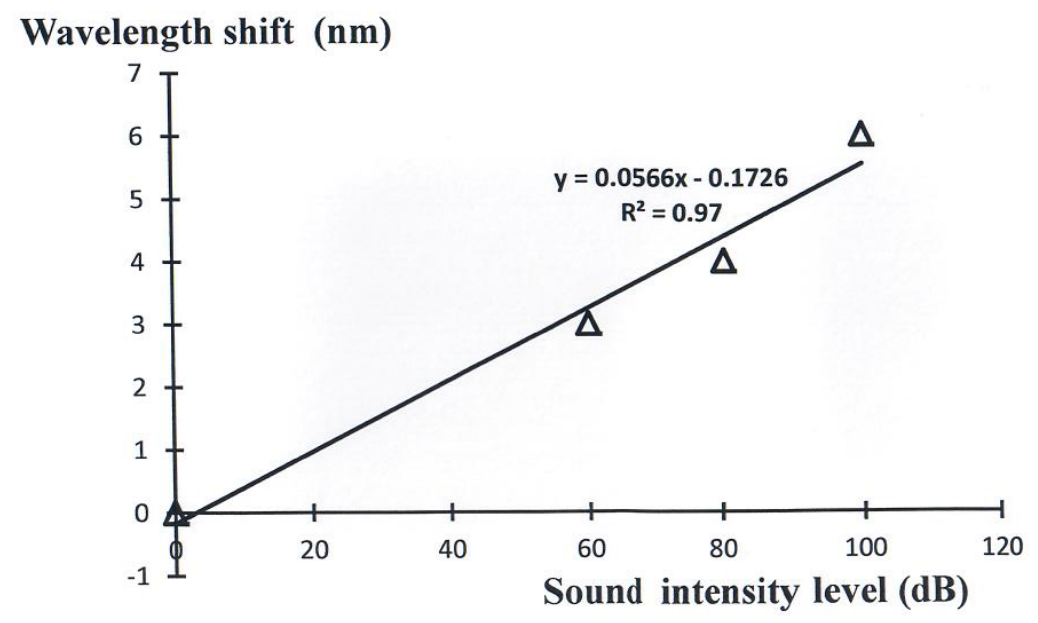

Fig. 6. Bragg wavelength shift $\left(\Delta \lambda_{B}\right)$ against applied sound intensity level $\left(I_{\text {sound }}\right)$.

\section{CONCLUSION}

An acoustic sensor based on FBG was designed and demonstrated. Strain due to sound intensity are able to induce changes in refractive index and the pitch of the grating. The results revealed that the designed sensor has quite linearity in the Bragg wavelength shift with sound intensity level. The sensitivity of sensor was $0.0566 \mathrm{~nm} / \mathrm{dB}$.

For future work, we purpose to enhance the sensitivity of sensor and apply it to monitor seismic wave, depth of under water pressure, and ultrasound detection. We suggest to use of appropriate coating or membrane attached on FBG in order to improve sensitivity and stability of the sensor.

\section{ACKNOWLEDGMENTS}

The present research was supported by grants from Faculty of Science, Silpakorn University, Thailand. The authors would like to thank Dr. Armote Somboonkaew and Thailand's National Electronics and Computer Technology Center (NECTEC) for supporting equipment and valuable technical suggestions.

\section{REFERENCES}

[1] M. M. Werneck, C. S. B. Allil, B. A. Ribeiro and F. V. B. De Nazaré, Current Trends in Short-and Long-Period Fibre Gratings; InTech: Rijeka, Croatia (2013) 1.

[2] H.-1. Liu, Z.-w. Zhu, Y. Zheng, B. Liu and F. Xiao, Optical Fiber Technology 40 (2018) 144.

[3] C. Li, X. Peng, H. Zhang, C. Wang, S. Fan and S. Cao, Measurement 117 (2018) 252.

[4] M.-f. Liang, X.-q. Fang, G. Wu, G.-z. Xue and H.-w. Li, Optik 145 (2017) 503.

[5] Z. Zhang, C. Shen and L. Li, Optics Communications 411 (2018) 108. 